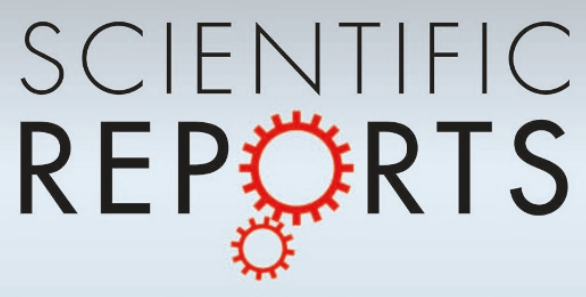

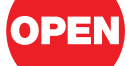

SUBJECT AREAS: GELS AND HYDROGELS MOLECULAR SELF-ASSEMBLY

APOPTOSIS

PROTEASES

Received

16 January 2013

Accepted

30 April 2013

Published

15 May 2013

Correspondence and requests for materials should be addressed to G.L.L. Igliang@ustc. edu.cn)

\section{DEVD-Based Hydrogelator Minimizes Cellular Apoptosis Induction}

\author{
An-Ming Tang ${ }^{1}$, Wei-Juan Wang ${ }^{1}$, Bin Mei ${ }^{1}$, Wang-Lai Hu${ }^{2}$, Mian Wu ${ }^{2} \&$ Gao-Lin Liang ${ }^{1}$
}

'CAS Key Laboratory of Soft Matter Chemistry, Department of Chemistry, University of Science and Technology of China, 96 Jinzhai Road, Hefei, Anhui 230026, China, ${ }^{2}$ School of Life Sciences, University of Science and Technology of China, Hefei, Anhui 230027, China.
Herein, we report the rational design of a DEVD-based heptapeptide hydrogelator 1 which is susceptible to caspase-3 (CASP3), and its isomeric control hydrogelator 2 with a DEDV-based heptapeptide sequence. Self-assembly of 1 in water results in flexuous, long nanofibers to form supramolecular hydrogel I with higher mechanical strength than that of hydrogel II which is composed of rigid, short nanofibers of 2 . In vitro enzymatic analysis indicated that 1 is susceptive to CASP3 while 2 is not. 3-(4,5-dimethylthiazol-2-yl) 2,5 diphenyl tetrazolium bromide (MTT) and Western blot analyses indicated that DEDV-based hydrogelator 2 induces cell death via apoptotic pathway while the DEVD-based hydrogelator 1 minimizes cellular apoptosis induction.
S upramolecular hydrogels, composed of small amount of (normally less than 5\%) three-dimensional (3D) networks (e.g., nanofibers) which gel large amount of water (more than 95\%), have attracted broad research interest and been extensively explored in recent years due to their inherent properties such as biocompatibility and biodegradability ${ }^{1-9}$. As one type of biomaterials, supramolecular hydrogels have exhibited promising applications for controlled drug release and delivery, biosensing, tissue engineering, and wound healing ${ }^{10-14}$. An important feature of supramolecular hydrogels is that their components (e.g., nanofibers) are resulted from the self-assembly of the building blocks (i.e., small molecules) via non-covalent interactions upon external perturbations. Compared with physical or chemical perturbations (e.g., $\mathrm{pH}$, temperature, ionic strength, ligand-receptor interactions, et al) for initiating the process of self-assembly ${ }^{15-18}$, enzymatic regulations (e.g., assembly or disassembly) associating with hydrogelation have drawn increasing attention because they integrate the processes with biological events ${ }^{19,20}$. There were a lot of examples of using enzymes to trigger the hydrogelations. For examples, $\mathrm{Xu}$ and co-workers have incorporated phosphatase/esterase with the intracellular hydrogelation to control the fate of cells ${ }^{21,22}$. Ulijn et al used thermolysin to catalyze self-assembly of nanofibers for immobilizing laminin to treat extracellular matrix (ECM) diseases ${ }^{23}$. However, only a few examples utilized enzymes to regulate the premade hydrogels for follow-up applications. Yang et al used phosphatase/kinase as an enzymatic switch to manipulate the gel/sol transition ${ }^{24}$. McNeil and Soellner designed thrombin-triggered gelation for protease detection and artificially mimicking blood clotting ${ }^{25}$. Tang and co-workers used caspase-3 (CASP3) to assist photolithography for spatial functionalization of hydrogels ${ }^{26}$.

Recently, researchers in the fields of tissue engineering and regeneration medicine have been particularly interested in 3D cell culture. Supramolecular hydrogels were widely chosen as the scaffolds to culture cells in three dimensions due to their biocompatibilities and biodegradabilities. CASP3, an important protease of caspase family, is tightly associated with cell apoptosis. A peptide sequence, DEVD, is a specific substrate for CASP3 cleavage $^{27-30}$. Therefore, we envisioned that hydrogels formed with DEVD-based molecular hydrogelators would probably exhibit better property for 3D cell culture than other supramolecular hydrogels because the DEVDbased hydrogelators might interact with CASP3 in cells and minimize cellular apoptosis induction which is very crucial for parent cells' passage on the scaffolds. To test this hypothesis, we designed several DEVD-based amphiphiles and identified a heptapeptide derivative Acetyl-Asp-Glu-Val-Asp-Gly-Gly-Gly-EDA-Fmoc (1), together with its isomeric control Acetyl-Asp-Glu-Asp-Val-Gly-Gly-Gly-EDA-Fmoc (2), could self-assemble into nanofibers and form supramolecular hydrogels in water thereafter. In contrast to the $49 \%$ of viability of HepG2 cells incubated with 2 at $400 \mu \mathrm{M}$ for 3 days, $86 \%$ of HepG2 cells treated with 1 at the same conditions survived. Western blot analysis indicated that the cells treated with 2 underwent apoptotic pathway while the apoptosis induction of those cells treated with $\mathbf{1}$ was minimized. We chose fluorenylmethyloxycarbonyl (Fmoc) as the hydrophobic part of the hydrogelators because many Fmoc-based amphiphiles including anti-inflammatory 
<smiles>CC(=O)N[C@@H](CC(=O)O)C(=O)NC(CCC(=O)O)C(=O)N[C@@H](C(=O)NC(CCC(=O)O)C(=O)NCC(=O)NCC(=O)NCC(=O)NCCNC(=O)OCC1c2ccccc2-c2ccccc21)C(C)C</smiles><smiles>CC(=O)N[C@@H](CC(=O)O)C(=O)NC(CCC(=O)O)C(=O)N[C@@H](CC(=O)O)C(=O)NC(C(=O)NCC(=O)NCC(=O)NCC(=O)NCCNC(=O)OCC1c2ccccc2-c2ccccc21)C(C)C</smiles>

Figure $1 \mid$ Chemical structures of hydrogelators 1 and 2.

agent NPC 15199 have been proven prone to self-assembling in water and resulting in supramolecular hydrogels ${ }^{31}$.

\section{Results}

Synthesis. We began the study with the syntheses of hydrogelators 1 and 2 . The syntheses of $\mathbf{1}$ and $\mathbf{2}$ are facile and straightforward as follows (Fig. 1 \& Supplementary Information): heptapeptides (AcDEVDGGG-OH (1b) for $\mathbf{1}$, or Ac-DEDVGGG-OH (2b) for 2) were synthesized with solid phase peptide synthesis (SPPS), then coupled with fluorenylmethyloxycarbonyl ethylenediamine (Fmoc-EDA), purified with high performance liquid chromatography (HPLC) to yield 1a or 2a respectively. Deprotection of 1a or 2a with $95 \%$ trifluoroacetic acid (TFA) in dichloromethane yields 1 or 2 after HPLC purification.

pH-controlled gelation of $1 \& 2$, and rheological characterizations of Gel I \& Gel II. After obtaining 1 and 2, we tested their gelation abilities. In brief, $3 \mathrm{mg}$ of 1 or 2 was suspended in $300 \mu \mathrm{L}$ of water (10.5 mM). Adjustment of the $\mathrm{pH}$ values of above suspensions to 11 with solid sodium carbonate resulted in clear solutions (upper panels of the insets in Fig. 2a\&b). Carefully adjusting the $\mathrm{pH}$ values of above solutions to 1 with $10 \mathrm{M} \mathrm{HCl}$ afforded opaque Gel I (lower panel of the insets in Fig. 2a) or Gel II (lower panel of the insets in Fig. 2b) respectively. To evaluate the viscoelastic properties of the gels, we firstly used dynamic strain sweep to determine the proper condition for the dynamic frequency sweep of Gel I and Gel II. As shown in
Fig. S2 in Supplementary Information, the values of the storage modulus $\left(\mathrm{G}^{\prime}\right)$ and the loss modulus $\left(\mathrm{G}^{\prime \prime}\right)$ of Gel I and Gel II exhibit a weak dependence from 0.1 to $1.0 \%$ of strain (with $G^{\prime}$ dominating $G^{\prime \prime}$ ), indicating that the samples are hydrogels. After setting the strain amplitude at $1.0 \%$ (within the linear response regime of strain amplitude), we used dynamic frequency sweep to study Gel I and Gel II. Fig. 2a shows that $G^{\prime}$ and $G^{\prime \prime}$ of Gel I slightly increase with the increase of frequency from 0.1 to $100 \mathrm{rad} \cdot \mathrm{s}^{-1}$. The values of $G^{\prime}$ are five times larger than those of $G^{\prime \prime}$ in the whole range $\left(0.1-100 \mathrm{rad} \cdot \mathrm{s}^{-1}\right)$, suggesting that Gel $\mathrm{I}$ is fairly tolerant to external force. For comparison, Fig. $2 b$ shows the $\mathrm{G}^{\prime}$ values of Gel II are five times larger than that of $\mathrm{G}^{\prime \prime}$ in the whole range $\left(0.1-100 \mathrm{rad} \cdot \mathrm{s}^{-1}\right)$, suggesting that Gel II is also tolerant to external force. Both $\mathrm{G}^{\prime}$ and $\mathrm{G}^{\prime \prime}$ values of Gel II are less than half of those of Gel I respectively, indicating that Gel I is elastically stronger than Gel II.

Fluorescence studies. Participation of the fluorenyl moiety of the Fmoc group in the gelation was investigated by measuring the fluorescence spectra of 1 and 2 with varying $\mathrm{pH}$ in water (Figure S3). The molecules were excited at $265 \mathrm{~nm}$. At dilute concentration $(1 \mathrm{mM}), \mathbf{1}$ and 2 were found to remain at the molecular state at $\mathrm{pH}$ values of 11,7 , and 4 , proven by the emission peaks at $313.5 \mathrm{~nm}$. When the $\mathrm{pH}$ values were lowered down to 1 , for both hygelators, obvious redshifted emissions were observed at $\lambda_{\mathrm{em}} 317 \mathrm{~nm}$, which confirms the self-assembly of the fluorenyl moiety with their aromatic rings being overlapped in parallel orientation.
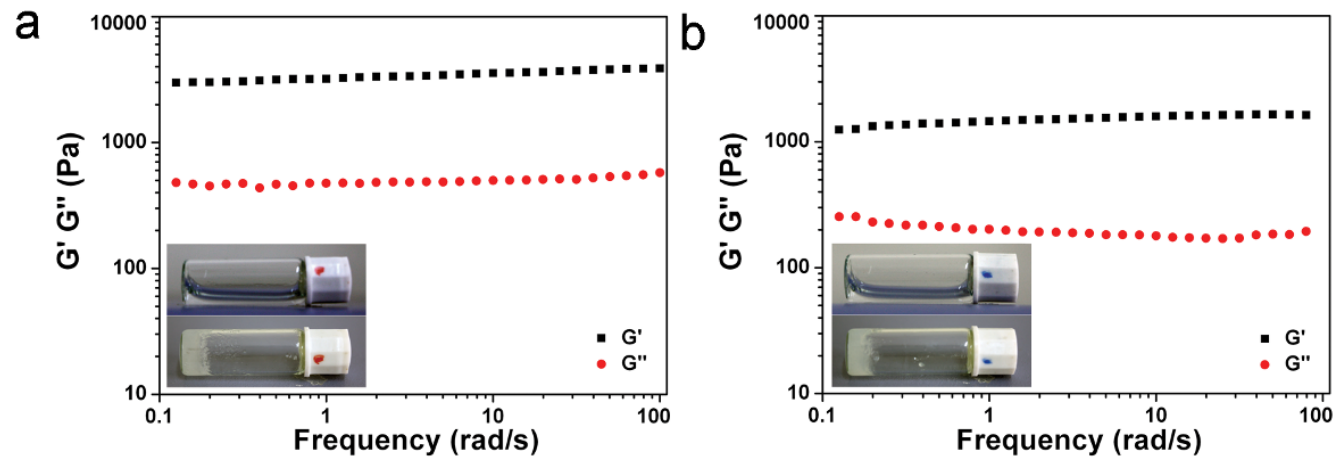

Figure $2 \mid$ Optical images and rheological analysis of Gel I and Gel II. (a) Dynamic frequency sweep of the storage moduli $\left(\mathrm{G}^{\prime}\right)$ and the loss moduli $\left(\mathrm{G}^{\prime \prime}\right)$ of Gel $\mathbf{I}$ at the strain of $1.0 \%$ (insets: Water solution of $\mathbf{1}$ at $1 \mathrm{wt} \%$ and $\mathrm{pH} 11$ (upper), and corresponding Gel $\mathbf{I}$ at pH 1 (lower)). (b) Dynamic frequency sweep of the storage moduli $\left(\mathrm{G}^{\prime}\right)$ and the loss moduli $\left(\mathrm{G}^{\prime \prime}\right)$ of Gel II at the strain of $1.0 \%$ (insets: Water solution of $\mathbf{2}$ at 1 wt $\%$ and $\mathrm{pH} 11$ (upper), and corresponding Gel II at pH 1 (lower)). All rheological measurements were carried out at $37^{\circ} \mathrm{C}$ and $\mathrm{pH} 1$. 


\section{a}

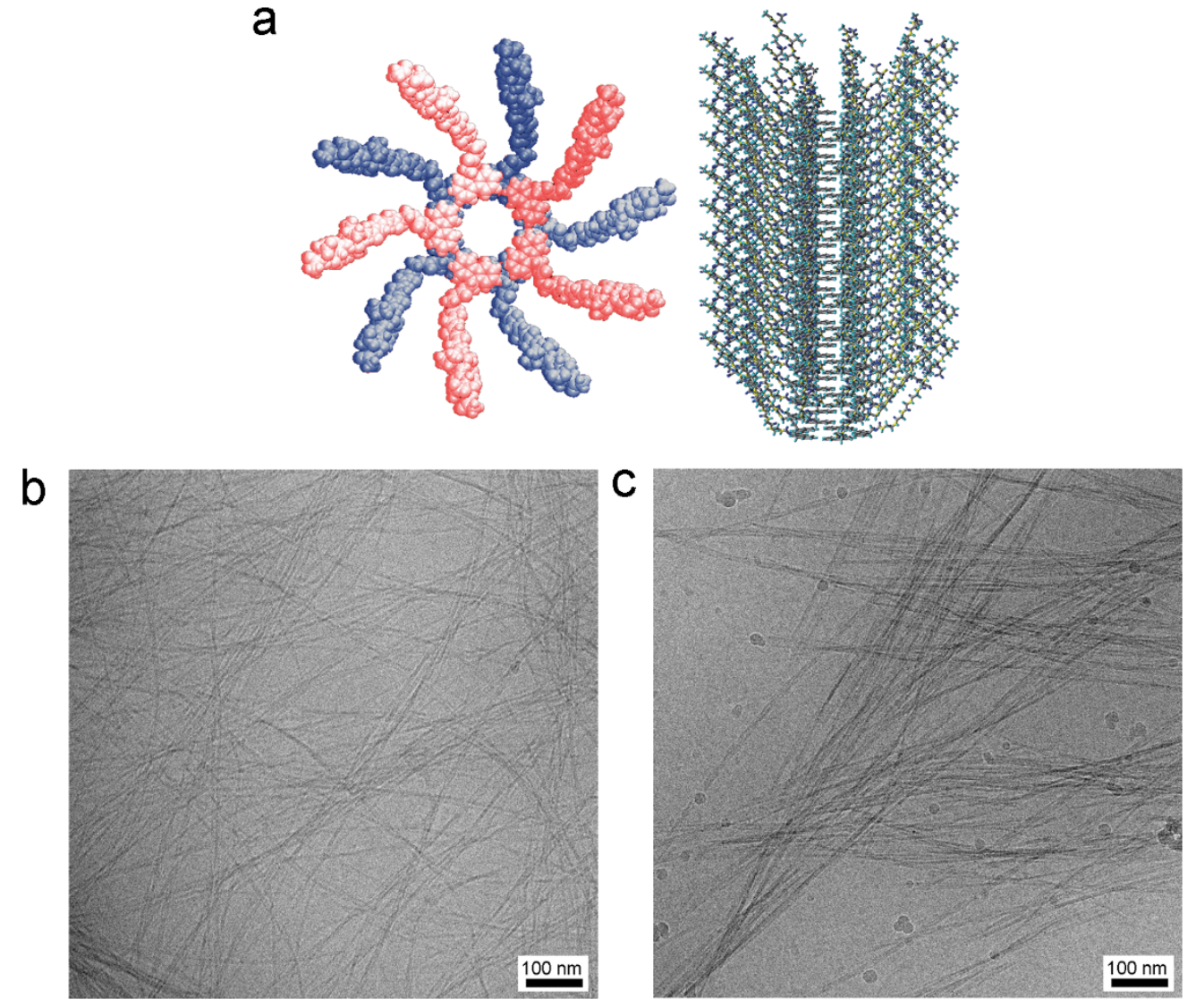

Figure 3 Proposed molecular arrangement of 1 in Gel I and morphological studies of Gel I and Gel II. (a) Hierarchical self-assembly of 1 into a five-membered supermacrocycle and resulting in nanofiber in Gel I, top (left) and side (right) views (VMD). (b) Cryo-TEM image of Gel I. c) Cryo-TEM image of Gel II.

Morphological studies of Gel I \& Gel II, and proposed molecular arrangement for Gel I. Cryo transmission electron microscopy (cryo-TEM) and atomic force microscopy (AFM) were employed to investigate the morphologies of Gel I and Gel II. The microscopic structure of Gel I under cryo-TEM exhibited flexuous, long fibers with an average width of $6.1 \pm 1.2 \mathrm{~nm}$ (Fig. 3b). AFM image of Gel I revealed that two or more fibers twist together during water evaporation at room temperature, resulting in bigger nanofibers with an average width of $23.7 \pm 4.1 \mathrm{~nm}$ (Supplementary Fig. S4a). CryoTEM image of Gel II showed rigid, short nanofibers with an average width of $7.7 \pm 2.2 \mathrm{~nm}$ (Fig. 3c). Its AFM image consistently showed short, rigid nanofibers with an average width of $41.1 \pm 12.8 \mathrm{~nm}$ (Supplementary Fig. S4b). According to the amphiphilic structure of the Fmoc-heptapeptide hydrogelator 1, a possible molecular arrangement was proposed for the nanofibers in Gel I obtained during gelation process in water. As shown in Fig. 3a, from top view, the nanofiber of Gel I is proposed to be cylindrical micellar structure formed by the self-assembly of 1 . In this model, molecules of 1 centripetally stack to form the hydrophobic inner tubular layer due to the strong $\pi-\pi$ interactions and the peptide segments of 1 stretch to the surrounding water to form the hydrophilic outer layer. Each molecule of $\mathbf{1}$ is placed under and between two molecules of 1 on the above layer with a layer distance of $0.45 \mathrm{~nm}$. Five molecules of 1 constitute one repeating unit of the tubular structure. Fourier transform infrared spectroscopy (FT-IR) analysis of Gel I indicates that the gel has a amide absorption at $1655 \mathrm{~cm}^{-1}$ redshifted from that of 1 at $1647 \mathrm{~cm}^{-1}$, suggesting the existence of large loop or $\alpha$-helical structures (Supplementary Fig. S5a) ${ }^{32}$. This is consistent with the AFM observation of Gel I (Supplementary Fig. S4a). For Gel II, the amide absorption at $1643 \mathrm{~cm}^{-1}$ remains unchanged compared with that of 2 , suggesting the structure of the nanofibers in Gel II is unordered (Supplementary Fig. S5b). This probably dues to the continuous DED peptide sequence of 2 breaking down $\alpha$-helical structure ${ }^{33}$, which is also consistent with the AFM observation of Gel II (Supplementary Fig. S4b). These findings satisfactorily explained why Gel I has better mechanical strength than that of Gel II (Fig. 2).

Enzymatic studies. After the characterizations of the hydrogels, we studied the susceptibility of hydrogelators 1 and 2 to CASP3. In principle, cleavage of the DEVD peptide sequence from 1 by CASP3 yields compound 3 and 4, as shown in Fig. 4a. After $100 \mathrm{~min}$ incubation of $0.28 \mathrm{mM}$ of 1 or 2 with CASP3 at $3.36 \mathrm{nmol} / \mathrm{U}$ and $37^{\circ} \mathrm{C}$, the incubation mixtures were directly injected into a HPLC system for analysis. HPLC trace of the incubation mixture of 1 clearly showed the disappearance of 1 at retention time of $15.3 \mathrm{~min}(1.8 \%)$ and the appearance of one new peak at retention time of $14.7 \mathrm{~min}$ (98.2\%, Fig. 4b). Electrospray ionization mass spectroscopic (ESIMS) analysis indicated that the new peak at $14.7 \mathrm{~min}$ is the enzy matic product of 1 which has characteristic absorbance at $300 \mathrm{~nm}$ (i.e., 4) after CASP3 cleavage (Supplementary Fig. S6). HPLC trace of the incubation mixture of 2 only exhibited one peak which has the same retention time of $15.5 \mathrm{~min}$ as that of 2 (Fig. $4 \mathrm{c}$ ). ESI-MS analysis of the peak indicated that it is 2, suggesting that 2 is not susceptive to CASP3 (Supplementary Fig. S7).

MTT assays and Western blot analyses. Prior to MTT assays, cell permeability of 1 and 2 were studied. As shown in Figure S8-10, after $2 \mathrm{~h}$ incubation with $200 \mu \mathrm{M}$ of 1 or 2 at $37^{\circ} \mathrm{C}$, HepG 2 cells were lysed and injected into HPLC for analysis. The results indicated that 1 was 
a
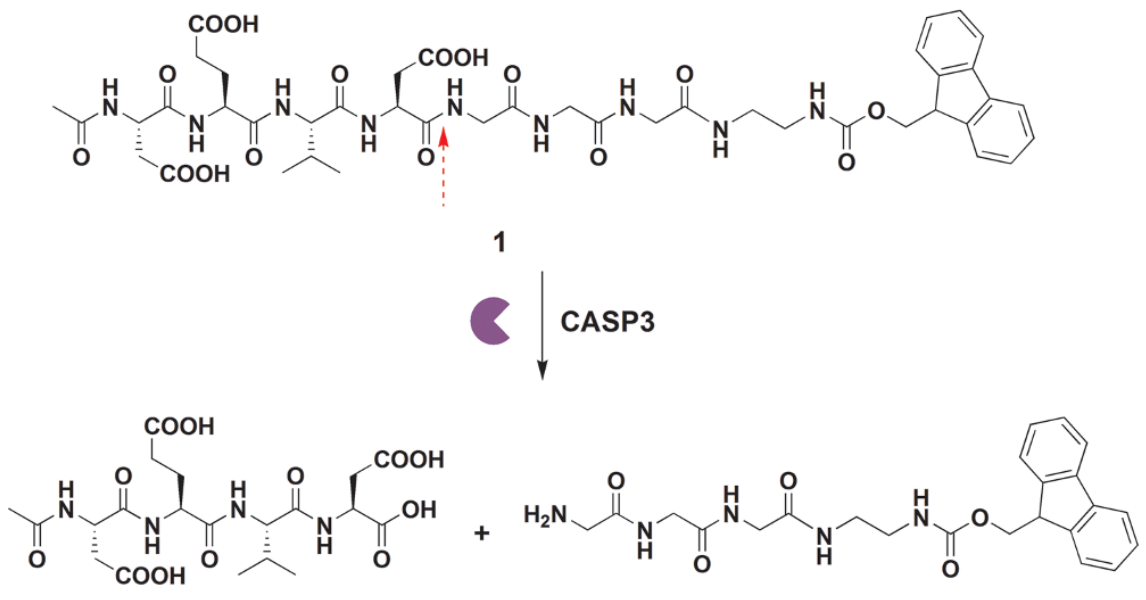

3

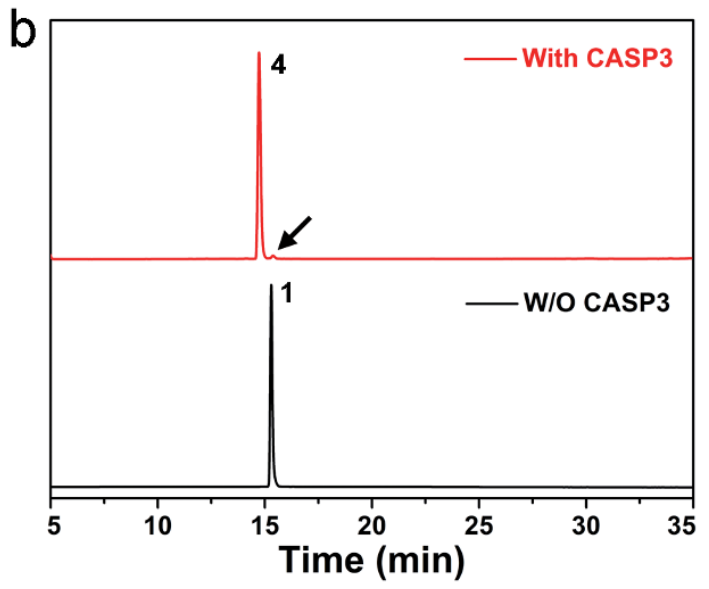

4

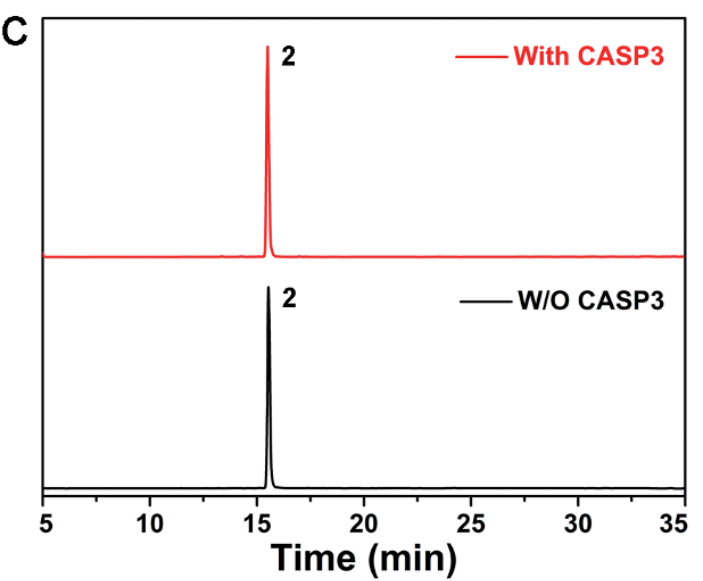

Figure $4 \mid$ HPLC analysis of enzyme degradability of $1 \& 2$ by CASP3. (a) Schematic illustration of enzymatic reaction of 1 with CASP3. (b) HPLC traces of $\mathbf{1}$ incubated with (red) or without (black) CASP3. Arrow indicates the small peak of $\mathbf{1}$. (c) HPLC traces of $\mathbf{2}$ incubated with (red) or without (black) CASP3. Wavelength for detection: $300 \mathrm{~nm}$.

uptaken by the cells at a final concentration of $0.40 \mathrm{fmol}$ per cell with $48.7 \%$ of 1 being metabolized and $51.3 \%$ of 1 not. As to 2, it was uptaken by the cells at a final concentration of $0.27 \mathrm{fmol}$ per cell with $37.1 \%$ of 2 being metabolized and $62.9 \%$ of 2 not. After the validation that 1 is susceptible to CASP 3 while 2 is not and both of these two hydrogelators have good cell permeability, we examined the cell viabilities of HepG2 cells in the presence of hydrogelator 1 or 2 at different concentrations using 3-(4,5-dimethylthiazol-2-yl) 2,5 diphenyl tetrazolium bromide (MTT) assay. As shown in Fig. 5a\&b, 1 obviously shows higher cellular viability than that of 2 . Detailedly, cells incubated with $200 \mu \mathrm{M}$ of 1 for 3 days exhibit $96.3 \%$ of viability (Fig. 5a). In contrast, only $82.0 \%$ of cells treated with $200 \mu \mathrm{M}$ of 2 survived at day 3 (Fig. 5b). At $400 \mu \mathrm{M}, 1$ still did not exhibit obvious cytotoxicity to the cells at day 3 ( $85.9 \%$ of cell viability, Fig. $5 \mathrm{a}$ ) while less than half (49.1\%) of the cells incubated with 2 survived (Fig. 5b). MTT assays of 1 and 2 on HeLa and MDA-MB-468 cells indicated that these two hydrogelators have cellular viabilities consistent with those on HepG2 cells (i.e., 1 has higher cellular viabilities than those of 2) (Supplementary Fig. S11\&12). To study the mechanism of the cell-viability difference between the cells treated with 1 and 2 , we harvested the cells after 3 -day incubation with $400 \mu \mathrm{M}$ of 1 or 2 and quantified the expressions of pro-caspase 3, activated CASP3 (i.e., cleaved-caspase 3), and cleaved poly(ADP-ribose) polymerase (PARP) using Western blot ${ }^{34-36}$. As shown in Fig. 5C, there was no obvious expression of cleaved-PARP in the cells treated with 1 . Moreover, these cells also did not show obvious expression of cleaved-caspase 3 but the expression of pro-caspase 3 in these cells was comparable to that in cells treated with $1 \%$ DMSO in culture medium (NC group). This indicates that these cells have not launched cell apoptosis after being incubated with $400 \mu \mathrm{M}$ of 1 for 3 days. In contrast, cells treated with 2 exhibited obvious activation of CASP3 (i.e., bands of cleaved-caspase 3 and cleaved PARP on the blots) and died via the apoptotic pathway, similar to that of a well-established, camptothecin (CPT)-induced cell apoptotic model ${ }^{37}$.

\section{Discussion}

Interestingly, formation of Gel I was completed within 5 min while that of Gel II took more than $10 \mathrm{~min}$. This suggests that $\mathbf{1}$ is more prone to self-assembling than 2, proven by the nanfibers in Gel I having narrower size-distribution than the nanofibers in Gel II. Both $\mathrm{G}^{\prime}$ and $\mathrm{G}^{\prime \prime}$ values of Gel I are twofold more of those of Gel II respectively. This also suggests that the Gel I composed of flexuous, long nanofibers of $\mathbf{1}$ has higher mechanical strength than that of Gel II which is composed of rigid, short nanofibers of 2 .

From the molecular arrangement model we proposed, the nanofiber in Gel I has a calculated width of $7.0 \mathrm{~nm}$, which fits well with our cryo-TEM observation (i.e., $6.1 \mathrm{~nm}$ ). FT-IR analysis of Gel I after water evaporation at room temperature indicates the existence of large loop or $\alpha$-helical structures which is consistent with the AFM image of Gel I clearly showing two or more fibers of 1 twist together resulting in bigger nanofibers at the same condition. In contrast, both FT-IR and AFM analyses of Gel II after water evaporation indicate that fibers in the gel are unordered structures. These also 
a

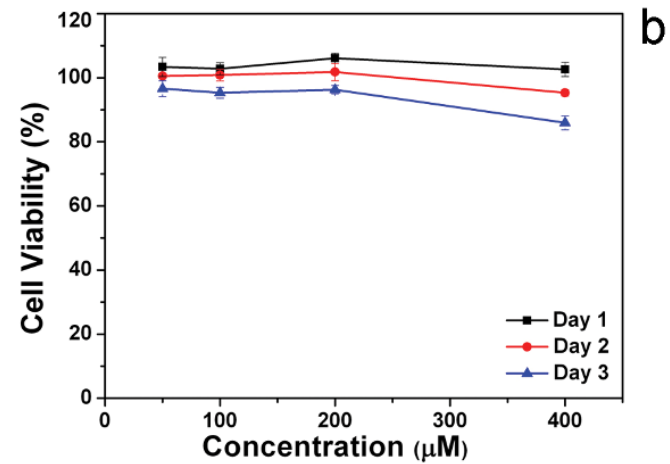

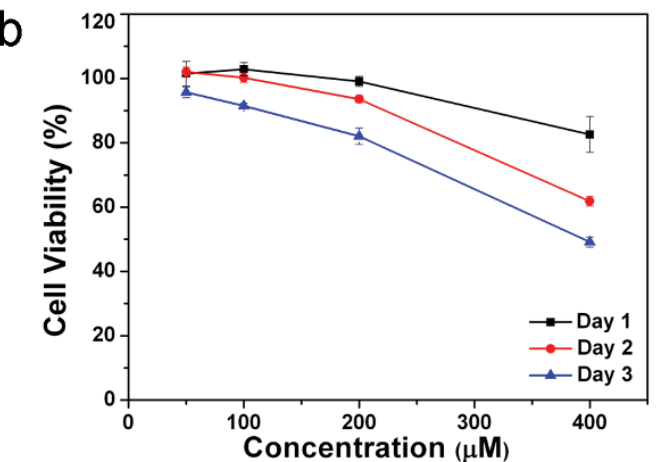

C

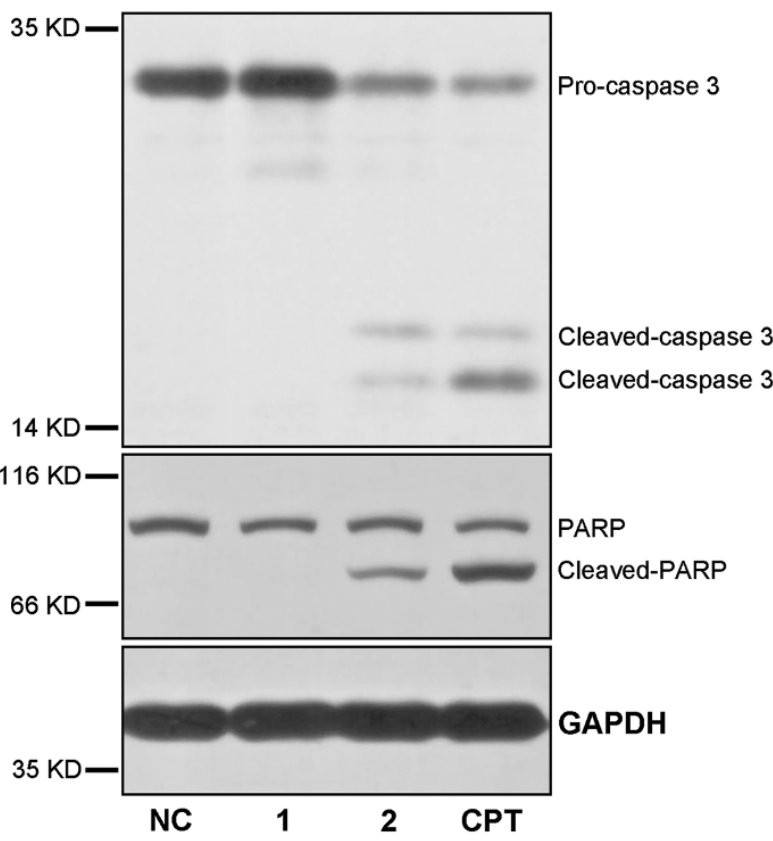

Figure 5 | Cytotoxicity tests and Western blot analyses of 1 and 2 on HepG2 cells. MTT assay of $\mathbf{1}$ (a) and 2 (b) on HepG2 cells. (c) Western blot analysis of expression of CASP3 and PARP in HepG2 cells incubated with 1\% DMSO (negative control, NC), $400 \mu \mathrm{M}$ of $\mathbf{1}$ or $\mathbf{2}$ for $72 \mathrm{~h}$, or with $10 \mu \mathrm{M}$ of $\mathrm{CPT}$ in culture medium for $6 \mathrm{~h}$.

satisfactorily explain why Gel I has higher mechanical strength than that of Gel II.

Enzymatic study of 1 incubated with CASP3 indicated that CASP3 has a twofold faster cleavage speed for $1(2 \mathrm{nmol} / \mathrm{U}$ per hour $)$ than for catalog substrate DEVD-pNA (1 nmol/U per hour), suggesting the DEVD $\downarrow$ G substrate designed herein is more efficient for CASP3 cleavage. In contrast, the control 2, isomer of 1 with VD being switched to DV, is indeed inactive to CASP3.

The big difference of cell viabilities between the cells treated with 1 and those with 2 could be ascribed to the structural difference between 1 and 2 . Detailedly, since 1 has a DEVD peptide sequence for CASP 3 cleavage, long time of incubation of the cells with 1 at high concentration might affect the activation or activity of CASP 3 which is responsible for cell apoptosis. In contrast, the DEDV peptide sequence of 2 is irresponsive to CASP3. Thus, 2 should not affect the apoptotic pathway induced by CASP3. Quantifications of the expression of cleaved-PARP in the Western blot indicated that cells treated with 1 only had a relative apoptotic level of $14 \%$ while that of cells treated with 2 was $58 \%$, comparing with that of CPT-induced apoptotic cells respectively (Supplementary Fig. S13). Interestingly, these quantitative results of Western blot (14\% of 1 and $58 \%$ of 2 ) are well complementary to the MTT results of the cells ( $86 \%$ of 1 and $49 \%$ of 2 ), suggesting that the cells treated with 1 or 2 are subjected to a caspase-guided apoptotic pathway.
In conclusion, by rational design of a DEVD-based, heptapeptide hydrogelator 1 and its isomeric control 2, we successfully obtained two supramolecular hydorgels Gel I and Gel II. Gel I is composed of flexuous, long nanofibers whose structure could be well simulated by a model of molecular arrangement of $\mathbf{1}$. Gel II is composed of short, rigid nanofibers and its mechanical strength is weaker than that of Gel I. Enzymatic tests indicated that 1 is susceptible to CASP 3 while 2 is not. MTT and Western blot analyses indicated 2 induces cell death via apoptotic pathway while 1 minimizes the cellular apoptosis induction. Structural optimization of $\mathbf{1}$ to form hydrogel at physiological condition for potential $3 \mathrm{D}$ cell culture and tissue engineering, this work is underway.

\section{Methods}

General methods. All the starting materials were obtained from Adamas or Sangon Biotech. Commercially available reagents were used without further purification, unless noted otherwise. All other chemicals were reagent grade or better. Active recombinant mouse CASP3 was obtained from BioVision as lyophilized powder ( $~ 300,000 \mathrm{U} / \mathrm{mg}, 1 \mathrm{U}$ corresponds to the amount of enzyme which cleaves $1 \mathrm{nmol}$ of the caspase substrate DEVD-pNA per hour at $37^{\circ} \mathrm{C}$ in a reaction solution containing $50 \mathrm{mM}$ Hepes, pH 7.2, $50 \mathrm{mM} \mathrm{NaCl}, 0.1 \%$ Chaps, $10 \mathrm{mM}$ EDTA, 5\% Glycerol, and $10 \mathrm{mM}$ TCEP). All the enzymatic studies were performed in CASP3 reaction solution described above. HPLC analyses were performed on an Agilent 1200 HPLC system equipped with a G1322A pump and an in-line diode array UV detector using an Agilent Zorbax 300SB- $\mathrm{C}_{18}$ RP column or an Extend- $\mathrm{C}_{18} \mathrm{RP}$ column with $\mathrm{CH}_{3} \mathrm{OH}$ $(0.1 \%$ of TFA $)$ or $\mathrm{CH}_{3} \mathrm{CN}(0.1 \%$ of TFA $)$ and ultrapure water $(0.1 \%$ of TFA $)$ as the 
eluent. The spectra of electrospray ionization-mass spectrometry (ESI-MS) were recorded on a LCQ Advantage MAX ion trap mass spectrometer (Thermo Fisher). ${ }^{1} \mathrm{H}$ NMR and ${ }^{13} \mathrm{C}$ NMR spectra were obtained on a $300 \mathrm{MHz}$ Bruker AV 300. AFM images were recorded on a Veeco multimode V system with the tip of Veeco RTESP. Rheology tests were operated on an AR 2000 ex (TA instrument) system, $40 \mathrm{~mm}$ parallel plates were used during the experiment at the gap of $300 \mu \mathrm{m}$. UV-vis absorbance spectra were recorded on a U-2910 UV-visible spectrophotometer (Hitachi High-Techonologies Corporation, Japan) at room temperature. $\mathrm{PH}$ dependent fluorescence emissions of 1 or 2 were recorded on an F-2500 fluorescence spectrophotometer (Hitachi High-Techonologies Corporation, Japan) at room temperature with excitation wavelength at $265 \mathrm{~nm}$. Cryo transmission electron micrographs (Cryo-TEM) were obtained on a Tecnai F20 transmission electron microscope (FEI), operating at $100 \mathrm{kV}$. The cryo-samples were prepared as following: a special copper grid coated with carbon was put into Gatan SOLARUSTM plasmacleaning system to remove hydrocarbon contamination on the sample holder, then the sample was dropped on the copper grid in FEI Vitrobot ${ }^{\mathrm{TM}}$ sample plunger. The sample preparation was completed in the plunger. MTT assays were carried out on a Varioskan Flash Spectral Scanning Multimode Reader (Thermo Fisher Scienfitic, Finland) using the absorbance at $570 \mathrm{~nm}$ and $680 \mathrm{~nm}$.

Cell uptake. The hepatocellular carcinoma HepG2 cells were incubated with $200 \mu \mathrm{M}$ of $\mathbf{1}$ or $\mathbf{2}$ in Dulbecco's Modified Eagle Medium (DMEM) (HyClone, Thermo Fisher Scienfitic, United States) supplemented with $10 \%$ fetal bovine serum (Sijiqing Biological Engineering Materials, Hangzhou, China) at $37^{\circ} \mathrm{C}$ for $2 \mathrm{~h}$. Then the cells were harvested, lysed, and injected into HPLC system for analysis.

Cytotoxicity test. The hepatocellular carcinoma HepG2 cells and cervical cancer HeLa cells were obtained from Department of Biochemistry and Molecular Biology, Anhui Medical University. The Human Breast cancer MDA-MB-468 cells were obtained from the Cancer Center of Sun Yat-sen University. HepG2 cells, HeLa cells, or MDA-MB-468 cells were seeded into a 96-well plate (HyClone, Thermo Fisher Scienfitic, United States) at a density of $3 \times 10^{3}$ per well in $100 \mu \mathrm{L}$ DMEM supplemented with $10 \%$ fetal bovine serum. After the cells were incubated at $37^{\circ} \mathrm{C}$ and $5 \% \mathrm{CO}_{2}$ for $24 \mathrm{~h}, 1$ or 2 was added at final concentrations of $50,100,200$, or $400 \mu \mathrm{M}$ respectively. Then the cells were incubated for another 24,48 , or $72 \mathrm{~h} .10 \mu \mathrm{L}$ per well of MTT solution ( $5 \mathrm{mg} \cdot \mathrm{ml}^{-1}$ PBS, Sangon Biotech, Shanghai, China) were added and incubated for $4 \mathrm{~h}$. Then the formazan crystals were dissolved with $100 \mu \mathrm{L}$ of $10 \%$ SDS in PBS. The optical density (OD) of the dissolved formazan crystals was measured at wavelengths of $570 \mathrm{~nm}$ and $680 \mathrm{~nm}$. The percentage of cell viability was calculated using the following equation: viability $(\%)=\mathrm{OD}_{\text {treatment group }} \times 100 / \mathrm{OD}_{\text {control group, }}$ where $\mathrm{OD}=\mathrm{OD}_{570-680 \mathrm{~nm}}$.

Western blot analysis. Western blot was performed as described by Mei et al. ${ }^{36,37}$. HepG2 cells were treated with $400 \mu \mathrm{M}$ of 1 or 2 for $72 \mathrm{~h}$, or camptothecin (CPT) for $6 \mathrm{~h}$. The following antibodies were used from the indicated sources: the primary antibody against glyceraldehyde-3-phosphate dehydrogenase (GAPDH) (FL-335, Santa Cruz) is a rabbit polyclonal antibody raised against amino acids 1-335 representing full length GAPDH of human origin, primary antibody against poly ADP-ribose polymerase (PARP) (F-2, Santa Cruz) is a mouse monoclonal antibody raised against amino acids 764-1014 mapping at the C-terminus of PARP of human origin, primary antibody against Caspase-3 (pAb, Stressgen) is a rabbit polyclonal antibody against recombinant human Caspase- 3 protein (full length), Alkaline phosphatase (AP)-conjugated secondary antibodies against mouse and rabbit IgG (Promega). Blots were developed by Thermo Western Blot stabilized substrate and images of the blots were obtained using Image Quant LAS-4000 (Fujifilm, Japan).

1. Silva, G. A. et al. Selective Differentiation of Neural Progenitor Cells by HighEpitope Density Nanofibers. Science 303, 1352-1355 (2004).

2. Ulijn, R. V. \& Woolfson, D. N. Peptide and protein based materials in 2010: From design and structure to function and application. Chem. Soc. Rev. 39, 3349-3350 (2010)

3. Kiyonaka, S. et al. Semi-wet peptide/protein array using supramolecular hydrogel. Nat. Mater. 3, 58-64 (2004).

4. Gao, Y., Shi, J., Yuan, D. \& Xu, B. Imaging enzyme-triggered self-assembly of small molecules inside live cells. Nat. commun. 3, 1033 (2012).

5. Yang, Z., Liang, G. \& Xu, B. Enzymatic hydrogelation of small molecules. Acc. Chem. Res. 41, 315-326 (2008).

6. Li, X. et al. Supramolecular nanofibers and hydrogels of nucleopeptides. Angew. Chem. Int. Ed. 50, 9365-9369 (2011).

7. Zhang, X. et al. Rational design of a tetrameric protein to enhance interactions between self-assembled fibers gives molecular hydrogels. Angew. Chem. Int. Ed. 51, 4388-4392 (2012).

8. Hirst, A. R., Escuder, B., Miravet, J. F. \& Smith, D. K. High-Tech Applications of Self-Assembling Supramolecular Nanostructured Gel-Phase Materials: From Regenerative Medicine to Electronic Devices. Angew. Chem. Int. Ed. 47, 8002-8018 (2008).

9. Wang, Q. et al. High-water-content mouldable hydrogels by mixing clay and a dendritic molecular binder. Nature 463, 339-343 (2010).

10. Liang, G. et al. Supramolecular Hydrogel of ad-Amino Acid Dipeptide for Controlled Drug Release in Vivo. Langmuir 25, 8419-8422 (2009).
11. Gao, Y., Zhao, F., Wang, Q., Zhang, Y. \& Xu, B. Small peptide nanofibers as the matrices of molecular hydrogels for mimicking enzymes and enhancing the activity of enzymes. Chem. Soc. Rev. 39, 3425-3433 (2010).

12. Jayawarna, V. et al. Nanostructured hydrogels for three-dimensional cell culture through self-assembly of fluorenylmethoxycarbonyl-dipeptides. Adv. Mater. 18, 611-614 (2006).

13. Yang, Z. et al. D-glucosamine-based supramolecular hydrogels to improve wound healing. Chem. Commun. 843-845 (2007).

14. Liang, G. et al. Using Congo red to report intracellular hydrogelation resulted from self-assembly of small molecules. Chem. Commun. 4096-4098 (2007)

15. Adler-Abramovich, L. et al. Self-Assembled Organic Nanostructures with Metallic-Like Stiffness. Angew. Chem. Int. Ed. 49, 9939-9942 (2010).

16. Ariga, K., Mori, T. \& Hill, J. P. Control of nano/molecular systems by application of macroscopic mechanical stimuli. Chem. Sci. 2, 195-203 (2011).

17. de Loos, M., Friggeri, A., van Esch, J., Kellogg, R. M. \& Feringa, B. L. Cyclohexane bis-urea compounds for the gelation of water and aqueous solutions. Org. Biomol. Chem. 3, 1631-1639 (2005).

18. Lee, N. S. et al. Tunable dual-emitting shell-crosslinked nano-objects as singlecomponent ratiometric $\mathrm{pH}$-sensing materials. J. Mater. Chem. 21, 14193-14202 (2011).

19. Li, J. et al. D-amino acids boost the selectivity and confer supramolecular hydrogels of a nonsteroidal anti-inflammatory drug (NSAID). J. Am. Chem. Soc. 135, 542-545 (2013).

20. Spruell, J. M. \& Hawker, C. J. Triggered structural and property changes in polymeric nanomaterials. Chem. Sci. 2, 18-26 (2011).

21. Gao, J. et al. Enzyme promotes the hydrogelation from a hydrophobic small molecule. J. Am. Chem. Soc. 131, 11286-11287 (2009).

22. Yang, Z., Xu, K., Guo, Z. \& Xu, B. Intracellular enzymatic formation of nanofibers results in hydrogelation and regulated cell death. Adv. Mater. 19, 3152-3156 (2007).

23. Williams, R. J. et al. Enzyme-assisted self-assembly under thermodynamic control. Nat. Nanotechnol. 4, 19-24 (2009).

24. Yang, Z., Liang, G., Wang, L. \& Xu, B. Using a kinase/phosphatase switch to regulate a supramolecular hydrogel and forming the supramolecular hydrogel in vivo. J. Am. Chem. Soc. 128, 3038-3043 (2006).

25. Bremmer, S. C., Chen, J., McNeil, A. J. \& Soellner, M. B. A general method for detecting protease activity via gelation and its application to artificial clotting. Chem. Commun. 48, 5482-5484 (2012).

26. Gu, Z. \& Tang, Y. Enzyme-assisted photolithography for spatial functionalization of hydrogels. Lab Chip 10, 1946-1951 (2010).

27. Liang, G., Ren, H. \& Rao, J. A biocompatible condensation reaction for controlled assembly of nanostructures in living cells. Nat. Chem. 2, 54-60 (2010).

28. Chen, Y. \& Liang, G. Enzymatic Self-Assembly of Nanostructures for Theranostics. Theranostics 139-147 (2012).

29. Talanian, R. V. et al. Substrate specificities of caspase family proteases. J. Biol. Chem. 272, 9677-9682 (1997).

30. Cao, C. Y., Chen, Y., Wu, F. Z., Deng, Y. \& Liang, G. L. Caspase-3 controlled assembly of nanoparticles for fluorescence turn on. Chem. Commun. 47, 10320-10322 (2011).

31. Yang, Z. M., Gu, H. W., Zhang, Y., Wang, L. \& Xu, B. Small molecule hydrogels based on a class of antiinflammatory agents. Chem. Commun. 208-209 (2004)

32. Surewicz, W. K., Mantsch, H. H. \& Chapman, D. Determination of protein secondary structure by Fourier transform infrared spectroscopy: A critical assessment. Biochemistry 32, 389-394 (1993).

33. Cook, D. A. Relation Between Amino Acid Sequence and Protein Conformation J. Mol. Biol. 29, 167-171 (1967)

34. Jin, L. et al. MicroRNA-149*, a p53-responsive microRNA, functions as an oncogenic regulator in human melanoma. Proc. Natl. Acad. Sci. USA 108, 15840-15845 (2011)

35. Mei, Y., Hahn, A. A., Hu, S. \& Yang, X. The USP19 Deubiquitinase Regulates the Stability of c-IAP1 and c-IAP2. J. Biol. Chem. 286, 35380-35387 (2011).

36. Mei, Y., Du, W., Yang, Y. \& Wu, M. Puma*Mcl-1 interaction is not sufficient to prevent rapid degradation of Mcl-1. Oncogene 24, 7224-7237 (2005).

37. Mei, Y. et al. Noxa/Mcl-1 balance regulates susceptibility of cells to camptothecininduced apoptosis. Neoplasia 9, 871-881 (2007).

\section{Acknowledgments}

This work was supported by the National Natural Science Foundation of China (Grants 21175122 and 91127036), the Fundamental Research Funds for Central Universities (Grant WK2060190018), and Anhui Provincial Natural Science Foundation (Grant 1108085J17).

\section{Author contributions}

A.T. and W.W. contributed equally to this work. They did the synthetic, rheological, nanocharacterization, and enzymatic experiments. W.H. and B.M. did MTT experiments. W.H. carried out Western blot experiments. M.W. helped with the project design and manuscript preparation. G.L. designed this project and wrote the paper. 


\section{Additional information}

Supplementary information accompanies this paper at http://www.nature.com/ scientificreports

Competing financial interests: The authors declare no competing financial interests.
License: This work is licensed under a Creative Commons

Attribution-NonCommercial-NoDerivs 3.0 Unported License. To view a copy of this license, visit http://creativecommons.org/licenses/by-nc-nd/3.0/

How to cite this article: Tang, A. et al. DEVD-Based Hydrogelator Minimizes Cellular Apoptosis Induction. Sci. Rep. 3, 1848; DOI:10.1038/srep01848 (2013). 